\title{
Triple Helix of Scleroglucan in Dilute Aqueous Sodium Hydroxide
}

\author{
Toshio YANAKI, Takemasa KoJIMA, and Takashi NORISUYE* \\ Research Laboratory, Taito Co., Higashishiriike-shinmachi, \\ Nagata-ku, Kobe 653, Japan. \\ *Department of Macromolecular Science, Osaka University, \\ Toyonaka, Osaka 560, Japan.
}

(Received August 7, 1981)

\begin{abstract}
A sample of scleroglucan, a commercially available, water-soluble polysaccharide, and its sonicated fragment were investigated by chemical analysis and by light scattering and viscometry with water containing $0.01 \mathrm{~N}$ sodium hydroxide $(\mathrm{NaOH})$, dimethylsulfoxide (DMSO), and water + DMSO mixtures at $25^{\circ} \mathrm{C}$ as the solvents. From the chemical analysis, this polysaccharide was found to be a $\beta-1,3$-D-glucan consisting essentially of the same repeating units as found for schizophyllan. The light scattering and viscosity measurements yielded the following results: (1) The weight-average molecular weights $M_{w}$ of the two scleroglucan samples in $0.01 N$ $\mathrm{NaOH}$ are roughly three times as large as those in DMSO, (2) the radii of gyration $\left\langle S^{2}\right\rangle^{1 / 2}$ and the intrinsic viscosities $[\eta]$ in $0.01 N \mathrm{NaOH}$ are close to those of the rodlike triple helices of schizophyllan with the same $M_{w}$ in $0.01 N \mathrm{NaOH}$, (3) the values of $\left\langle S^{2}\right\rangle^{1 / 2} / M_{w}{ }^{1 / 2}$ and $[\eta] / M_{w}{ }^{1 / 2}$ for the scleroglucan samples in DMSO are approximately constant, and the second virial coefficients in this solvent are almost zero, and (4) $[\eta]$ of the sonicated sample in water + DMSO mixtures undergoes an almost discontinuous decrease at about $87 \mathrm{wt} \%$ DMSO at which the schizophyllan triple helix dissociates to single chains. Based on these results and structural information, it was concluded that the scleroglucan studied exists in $0.01 \mathrm{~N} \mathrm{NaOH}$ as a triple helix similar to that of schizophyllan in either pure water or $0.01 \mathrm{~N} \mathrm{NaOH}$, while it is dispersed in DMSO as a single chain and behaves like an unperturbed flexible chain.

KEY WORDS Polysaccharide / Scleroglucan / Schizophyllan / Triple Helix / Chemical Structure / Molecular Weight / Radius of Gyration / Intrinsic Viscosity /
\end{abstract}

Scleroglucan is the general name for capsular polysaccharides produced by species of the genus sclerotium including a fungus sclerotium glucanicum. One of these polysaccharides is commercially available under the trade name of Polytran (Ceca S. A., France). According to a technical report ${ }^{1}$ of Ceca S. A., this scleroglucan is water-soluble and nonionic.

A long time ago, Johnson et al. ${ }^{2}$ investigated the chemical structure of a scleroglucan from an unidentified species of sclerotium and concluded that this polysaccharide was a $\beta$-1,3-D-glucan consisting of the repeating units shown in Figure 1. These repeating units are exactly the same as those reported for schizophyllan,,$^{3,4}$ an extracellular polysaccharide produced by a fungus schizophyllum commune. Thus, scleroglucans are usually consider- ed to be the same polysaccharide as schizophyllan.

In this paper, we report a study made on a sample of Polytran scleroglucan and its sonicated fragment in order to examine whether scleroglucan is chemically identical with schizophyllan and whether these two polysaccharides show the same dimensional and hydrodynamic behavior in dilute solution. For the former problem, we applied the same methods of chemical analysis as those established for schizophyllan. ${ }^{4}$ For the latter problem, we determined the weight-average molecular weights, radii of gyration, and intrinsic viscosities of the two samples in $0.01 \mathrm{~N}$ sodium hydroxide $(\mathrm{NaOH})$ and dimethylsulfoxide (DMSO), in which schizophyllan dissolves as a rodlike triple 


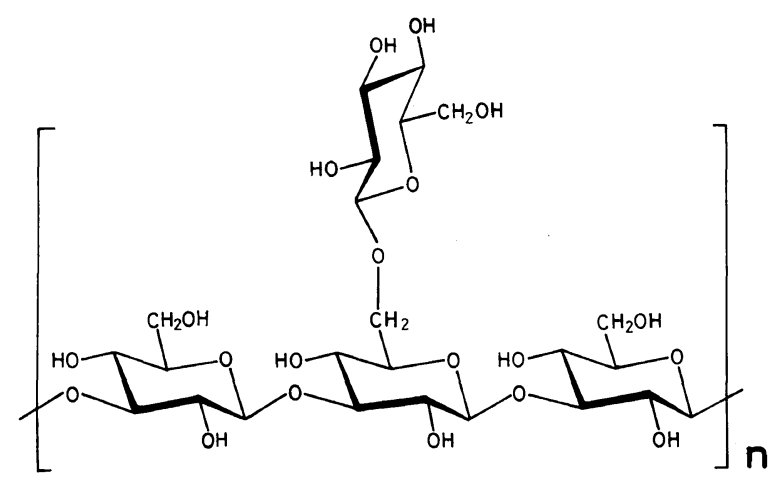

Figure 1. Repeat unit of scleroglucan. ${ }^{2}$

helix and a random coil, respectively. ${ }^{5,6} \mathrm{We}$ also compared the two polysaccharides in regard to the composition dependence of intrinsic viscosity in water + DMSO mixtures.

\section{EXPERIMENTAL}

\section{Samples}

A scleroglucan sample (Ceca S. A. Polytran R), supplied by Mitsui-Bussan Co., was purified by the method $^{4,5}$ established for schizophyllan. The purified sample (designated below as $\mathrm{N}$ ) dissolved completely in water, giving a perfectly transparent solution. Part of the sample $\mathrm{N}$ was sonicated by the method described elsewhere. ${ }^{4,5}$ The resulting fragment designated below as $\mathrm{S}$ and the sample $\mathrm{N}$ were each divided into three parts by fractional precipitation with water as the solvent and acetone, the precipitant. The middle fractions, designated below as $\mathrm{S}-2$ and $\mathrm{N}-2$, were chosen for the present study and freeze-dried from aqueous solutions.

\section{Chemical or Biochemical Analysis}

1. Analysis of Component Sugars. Each of the samples S-2 and N-2 $(10 \mathrm{mg})$ were hydrolyzed with $6 \mathrm{~N}$ sulfuric acid $\left(1 \mathrm{~cm}^{3}\right)$ for $1 \mathrm{~h}$ at $120^{\circ} \mathrm{C}$, and the hydrolysate was made neutral with barium carbonate. The turbid neutral solution was centrifuged, and the supernatant was passed through a column of Amberlite IR-120B and a membrane filter. After the eluate had been concentrated and brought to a $\mathrm{pH}$ higher than 8 by the addition of $2 N$ ammonium hydroxide, the sugars in the eluate were reduced with sodium borohydride $(50 \mathrm{mg})$ at room temperature. The excess borohydride was decomposed by adding acetic acid. To remove the residual borate, methanol $\left(2 \mathrm{~cm}^{3}\right)$ was added to the reaction mixture and allowed to evaporate at $40^{\circ} \mathrm{C}$ under reduced pressure. This procedure was repeated five times to ensure the complete removal of the borate. The resulting alditols were acetylated by heating with a $1: 1$ pyridine + acetic anhydride mixture $\left(0.5 \mathrm{~cm}^{3}\right)$ for $2 \mathrm{~h}$ at $100^{\circ} \mathrm{C}$.

The products were investigated by gas chromatography at $190^{\circ} \mathrm{C}$ using a $2 \mathrm{~m}$ column of $3 \%$ ECNSS M-Gaschrom Q. The results indicated that the two scleroglucan samples consisted only of D-glucoses.

2. Methylation. The samples S-2 and N-2 were methylated by the method of Hakomori. ${ }^{7}$ Each sample $\left(20 \mathrm{mg}\right.$ ) was dissolved in $2 \mathrm{~cm}^{3}$ DMSO under nitrogen atmosphere, and the resulting solution was treated with methylsulfinyl carbanion $\left(0.5 \mathrm{~cm}^{3}\right)$ for $4 \mathrm{~h}$ at room temperature and then with methyl iodide $\left(1.5 \mathrm{~cm}^{3}\right)$ for $2 \mathrm{~h}$ at $25^{\circ} \mathrm{C}$. The solution was diluted with $10 \mathrm{~cm}^{3}$ water and dialyzed against water. The whole methylation procedure was repeated until infrared absorption peaks characteristic of the hydroxyl groups of D-glucans disappeared.

The fully methylated glucans $(5 \mathrm{mg})$ were hydrolyzed with $90 \%$ formic acid $\left(0.4 \mathrm{~cm}^{3}\right)$ for $12 \mathrm{~h}$ at $100^{\circ} \mathrm{C}$ and heated with $2 \mathrm{M}$ trifluoroacetic acid $\left(0.5 \mathrm{~cm}^{3}\right)$ for $7 \mathrm{~h}$ at the same temperature. The methylated sugars were acetylated in the same way as described above. The products were subjected to gas chromatography.

3. Periodate Oxidation and Mild Smith Degradation. Each scleroglucan sample $(80 \mathrm{mg})$ was oxidized with $0.01 \mathrm{M}$ sodium metaperiodate $(50 \mathrm{mg})$ at $5^{\circ} \mathrm{C}$ in the dark. The consumption of periodate and the production of formic acid were determined as functions of time by the Fleury-Lange method ${ }^{8}$ and by titration with $0.01 \mathrm{~N} \mathrm{NaOH}$, respectively. 
After completion of the oxidation, ethylene glycol $\left(10 \mathrm{~cm}^{3}\right)$ was added and the mixture was dialyzed against water for $24 \mathrm{~h}$ at $5^{\circ} \mathrm{C}$.

The oxidized glucans were reduced with sodium borohydride, and the resulting glucan-polyalcohols were slowly hydrolyzed with $0.1 \mathrm{~N}$ sulfuric acid at room temperature (mild Smith degradation $^{9}$ ). The Smith-degraded glucans were water-insoluble. They were collected by centrifugation, washed thoroughly with water, dried in vacuo at $40^{\circ} \mathrm{C}$, and subjected to subsequent experiments.

4. Hydrolysis with Exo- $\beta$-1,3-Glucanase. Each of the Smith-degraded glucans $(20 \mathrm{mg})$ was dispersed in $1.5 \mathrm{~cm}^{3}$ of a Mcllvaine buffer $(\mathrm{pH} 4)$ at $50^{\circ} \mathrm{C}$ and hydrolyzed with an enzyme exo- $\beta$-1,3-glucanase prepared from a culture of Basidiomycetes QM$806 .{ }^{10}$ The reducing power of the solution was determined as a function of time by the SomogyiNelson method. ${ }^{11}$ The samples S-2 and N-2 were similarly hydrolyzed with exo- $\beta$-1,3-glucanase. All the hydrolysates were investigated by paper chromatography with Whatman No. 50 paper and 1buthanol + 2-propanol + water $(3: 12: 4)$ as the developing solvent.

\section{Light Scattering}

Light scattering measurements on the samples $\mathrm{S}-2$ and N-2 in $0.01 \mathrm{~N} \mathrm{NaOH}$ and DMSO at $25^{\circ} \mathrm{C}$ were made on a Fica 50 automatic light scattering photometer in an angular range from $22.5^{\circ}$ to $150^{\circ}$. Vertically polarized incident light of $436 \mathrm{~nm}$ wavelength was used for the sample S-2 in $0.01 \mathrm{~N} \mathrm{NaOH}$ and for the samples $\mathrm{S}-2$ and $\mathrm{N}-2$ in DMSO, and that of $546 \mathrm{~nm}$ was used for the sample N-2 in $0.01 \mathrm{~N}$ $\mathrm{NaOH}$. The experimental procedures were the same as those employed by Kashiwagi et al. ${ }^{6}$ in their study of schizophyllan in $0.01 \mathrm{~N} \mathrm{NaOH}$ and DMSO.

The specific refractive index increments of scleroglucan in $0.01 \mathrm{~N} \mathrm{NaOH}$ at $25^{\circ} \mathrm{C}$ were 0.145 $\mathrm{cm}^{3} \mathrm{~g}^{-1}$ at $436 \mathrm{~nm}$ and $0.142 \mathrm{~cm}^{3} \mathrm{~g}^{-1}$ at $546 \mathrm{~nm}$ and those in DMSO at $25^{\circ} \mathrm{C}$ were $0.063 \mathrm{~cm}^{3} \mathrm{~g}^{-1}$ at both $436 \mathrm{~nm}$ and $546 \mathrm{~nm}$.

\section{Viscometry}

Zero shear-rate viscosities of the samples S-2 and $\mathrm{N}-2$ in $0.01 \mathrm{~N} \mathrm{NaOH}$ at $25^{\circ} \mathrm{C}$ were determined by a four-bulb capillary viscometer of the Ubbelohde type and those in DMSO at $25^{\circ} \mathrm{C}$ by a conventional capillary viscometer.
The composition dependence of intrinsic viscosity $[\eta]$ in water + DMSO mixtures at $25^{\circ} \mathrm{C}$ was determined for the sample $\mathrm{S}-2$, using capillary viscometers with no correction needed for shearrate effect. A weighed amount of the sample was dissolved in the water+DMSO mixture of a desired composition at a temperature below $25^{\circ} \mathrm{C}$.

\section{RESULTS}

\section{Chemical Structure}

Figure 2 shows the gas chromatograms of the acetylated products obtained from the methylation experiment. Each product has three peaks 1, 2, and 3 ; the peak at the shortest retention time corresponds to the solvent chloroform. From the retention times, ${ }^{12}$ peaks 1,2 , and 3 were identified as 1,5-di- $O$-acetyl- 2,3,4,6-tetra- $O$ - methyl- D- glucitol (A), 1,3,5-tri- $O$ - acetyl- 2,4,6- tri- $O$ - methyl- Dglucitol (B), and 1,3,5,6-tetra- $O$ - acetyl-2,4-di- $O$ methyl-D-glucitol (C), respectively. The molar

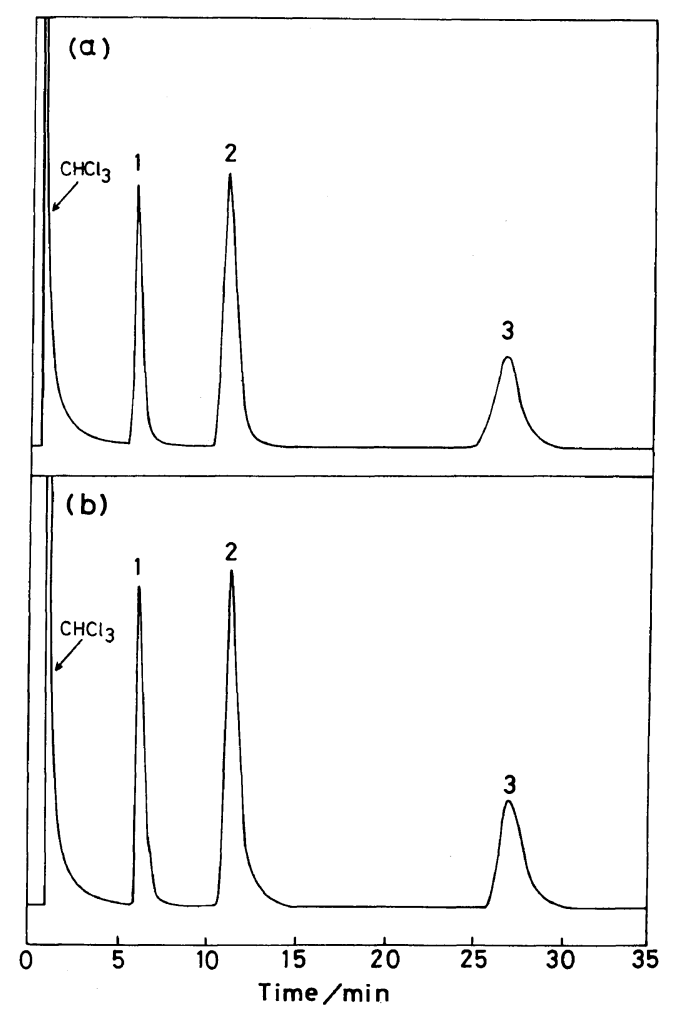

Figure 2. Gas chromatograms of the acetylated products obtained from methylation experiments: (a), sample N-2; (b), sample S-2. 
T. YANAKI, T. KoJIMA, and T. NorISUYE

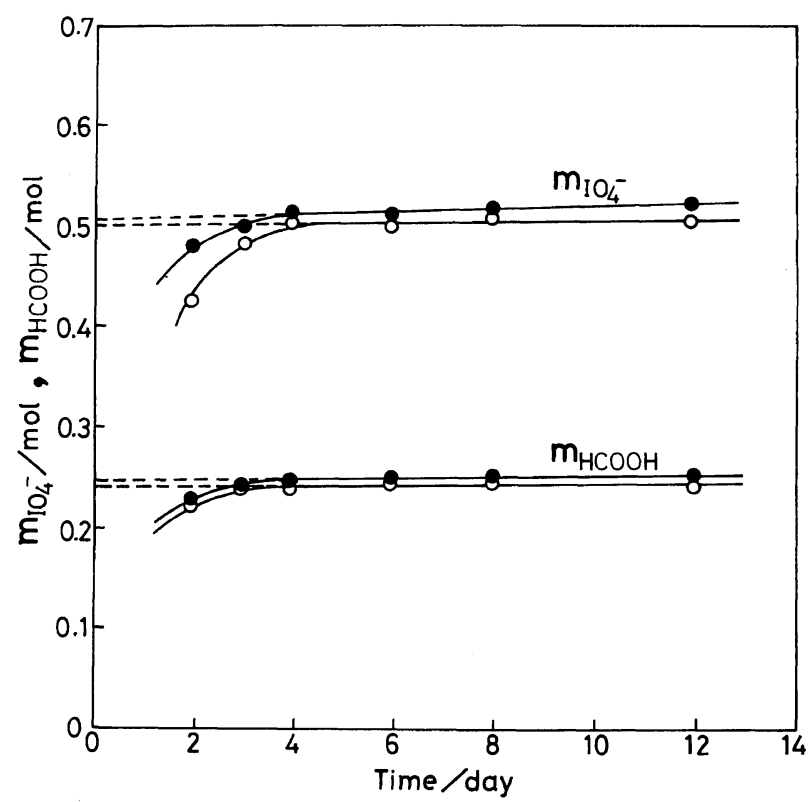

Figure 3. Amount of periodate $m_{\mathrm{IO}_{4}}$ - (moles per glucose residue) consumed and that of formic acid $m_{\mathrm{HCOOH}}$ produced by periodate oxidation of the scleroglucan samples $\mathrm{N}-2(\mathrm{O})$ and $\mathrm{S}-2(\bigcirc)$.

ratios of products $\mathrm{A}, \mathrm{B}$, and $\mathrm{C}$ evaluated from the area under the curves are $1.00: 1.99: 0.98$ for the sample $\mathrm{N}-2$ and $1.00: 2.00: 1.01$ for the sample $\mathrm{S}-2$. These ratios indicate that the repeat units of the two samples consist of three 1,3-linked Dglucose residues and one 1,6-linked D-glucose residue.

The results from the periodate oxidation experiment, displayed in Figure 3, show that each scleroglucan sample consumes $0.5 \mathrm{~mol}$ of periodate per glucose residue and produces $0.25 \mathrm{~mol}$ of formic acid. These values confirm the finding from the methylation experiment that one in every four glucose residues is a 1,6-linked glucopyranose.

Though not shown here, the paper chromatograms of the enzymatic hydrolysates from the Smith-degraded glucans revealed that only glucose was produced by exo- $\beta$-1,3-glucanase. The implication of this together with the result from the methylation or oxidation experiment is that since neither gentiobiose $\left(\mathrm{G}_{1} \frac{\beta}{6} \mathrm{G}\right)$ nor isomaltose $\left(G_{1} \alpha_{6} G\right)$ could be detected, our scleroglucan consists of repeat units below

$$
-{ }_{1} \mathrm{G}_{3}-{ }_{1}^{\mathrm{G}} \mathrm{G}_{3}^{6}-{ }_{1} \mathrm{G}_{3}-
$$

and that all the 1,3-linkages must be of the $\beta$-type.
Here, $\mathrm{G}$ denotes a D-glucose residue and the numbers attached to $G$ indicate the modes of linkage.

On the other hand, the paper chromatograms of the enzymatic hydrolysates from the samples S-2 and $\mathrm{N}-2$ showed the spot for gentiobiose as well as that for glucose. The molar ratio of glucose to gentiobiose was 1.96 for the sample S-2 and 2.08 for the sample $\mathrm{N}-2$ when estimated by the phenolsulfuric acid method of Dubois et al. ${ }^{13}$ This result confirms the repeat unit shown above, and moreover substantiates that the 1,6-linkage in the repeat unit is of the $\beta$-type. Thus, we conclude that the commercially available scleroglucan (Polytran) and its sonicated product examined in this work consist essentially of the same repeat unit as that of schizophyllan and also that of the scleroglucan studied by Johnson et al. ${ }^{2}$

From the chemical structure so established for our scleroglucan, we further conclude that the Smith-degraded glucan prepared from the sample $S$ 2 or $\mathrm{N}-2$ is a curdlan type $\beta-1,3-\mathrm{D}-$ glucan with no side chain. We determined its $[\eta]$ in a $1: 1$ water + triethylene diamine cadomium hydroxide (cadoxen) mixture to estimate the viscosity-average molecular weight $M_{v}$ using the $[\eta]-M_{w}$ (weightaverage molecular weight) relation established by Hirano et al. ${ }^{14}$ for curdlan in this mixed solvent. The $M_{v}$ value obtained was about $70 \%$ of 


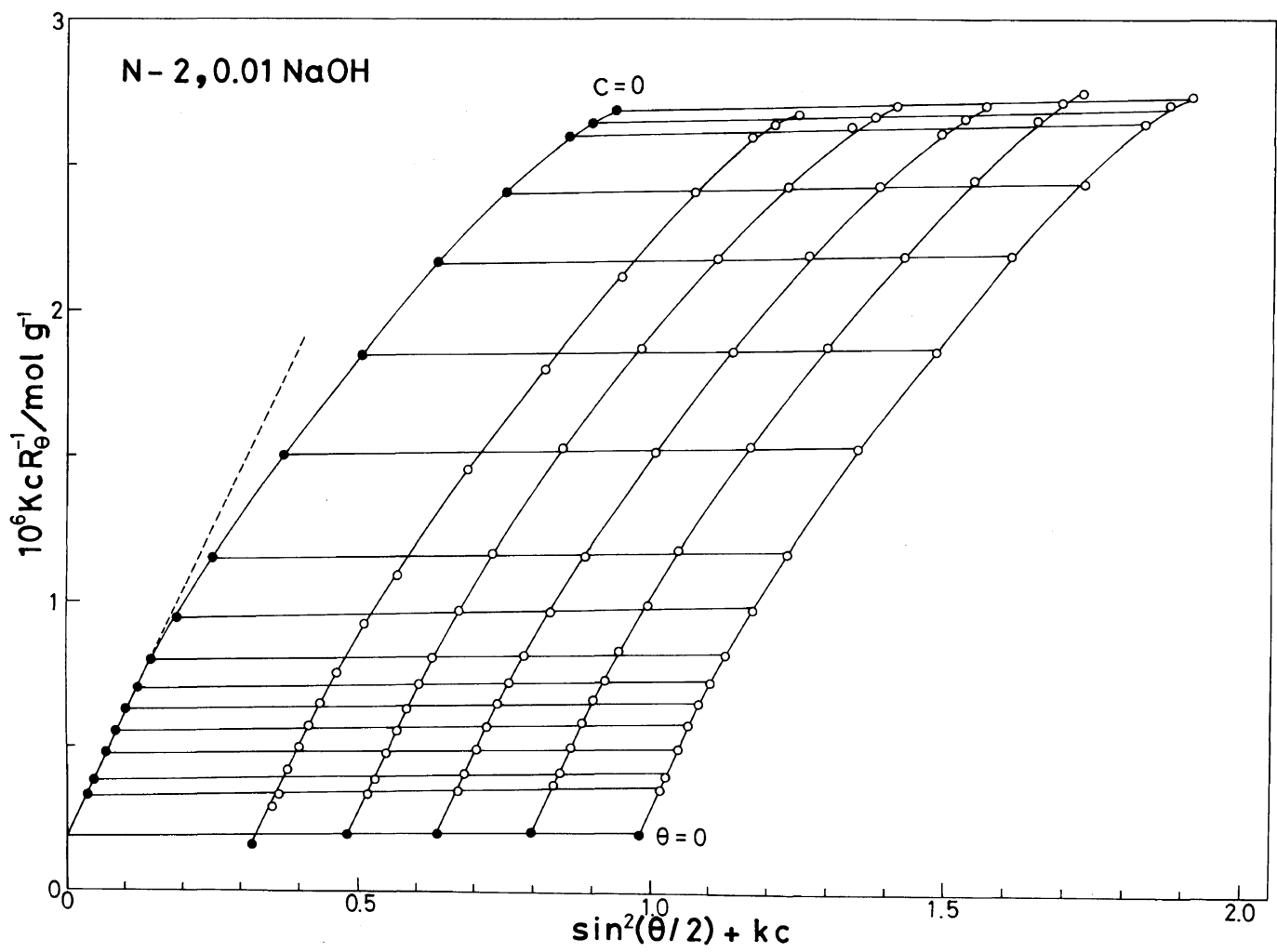

Figure 4. Zimm plot for the scleroglucan sample $\mathrm{N}-2$ in $0.01 \mathrm{~N} \mathrm{NaOH}$ at $25^{\circ} \mathrm{C}$. The constant $k$ is taken to be $5000 \mathrm{~cm}^{3} \mathrm{~g}^{-1}$. The dashed line indicates the initial slope of the curve for $c=0$.

the $M_{w}$ of the original scleroglucan sample $\mathrm{S}-2$ or $\mathrm{N}-2$ in DMSO (see Table I). This percentage is close to $75 \%$ which can be expected if the scleroglucan chain contains no $\beta$-1,6-linkage in the main chain, and supports the conclusion that the main chains of our scleroglucan samples contain only $\beta$-1,3-D-glucosidic linkages. Tabata et al. ${ }^{4}$ have used this viscosity method to confirm that the same is true for schizophyllan.

\section{Molecular Weight and Radius of Gyration}

Figure 4 illustrates the Zimm plot for the sample $\mathrm{N}-2$ in $0.01 N \mathrm{NaOH}$. Here, $K$ is the optical constant, $c$ the polymer mass concentration, and $R_{\theta}$ the reduced scattering intensity at scattering angle $\theta$. The values of $M_{w}, A_{2}$ (the second virial coefficient), and $\left\langle S^{2}\right\rangle^{1 / 2}$ (the radius of gyration) evaluated from the graph by the conventional method are presented in Table I, together with those for the same sample in DMSO and the sample S-2 in $0.01 N \mathrm{NaOH}$ and DMSO. The ratios of $M_{w}$ in $0.01 N \mathrm{NaOH}$ to $M_{w}$ in
DMSO calculated from the $M_{w}$ data are roughly 3 , indicating that the predominant species in an $0.01 \mathrm{~N}$ $\mathrm{NaOH}$ solution of scleroglucan is a trimer, as previously found to be the case with schizophyllan in $0.01 \mathrm{~N} \mathrm{NaOH}^{6}$ or in pure water. ${ }^{5}$

In Figure 5, the radius of gyration data for the samples S-2 and N-2 in $0.01 N \mathrm{NaOH}$ and DMSO are compared with those (the solid lines) obtained by Kashiwagi et al. ${ }^{6}$ for a series of schizophyllan samples in the same solvents. The points for $0.01 \mathrm{~N}$ $\mathrm{NaOH}$ fall close to the line for schizophyllan in the same solvent, suggesting that the scleroglucan trimer is a rodlike particle, almost the same as the schizophyllan trimer which has been shown to be modeled by a semiflexible rod with a very large persistence length of about $180 \mathrm{~nm} .^{6}$ The data points for DMSO lie appreciably below the line for schizophyllan in the same solvent. If these points are fitted by a straight line as indicated, a value of 0.5 is obtained for the slope. Since this slope is expected for unperturbed flexible coils, the 


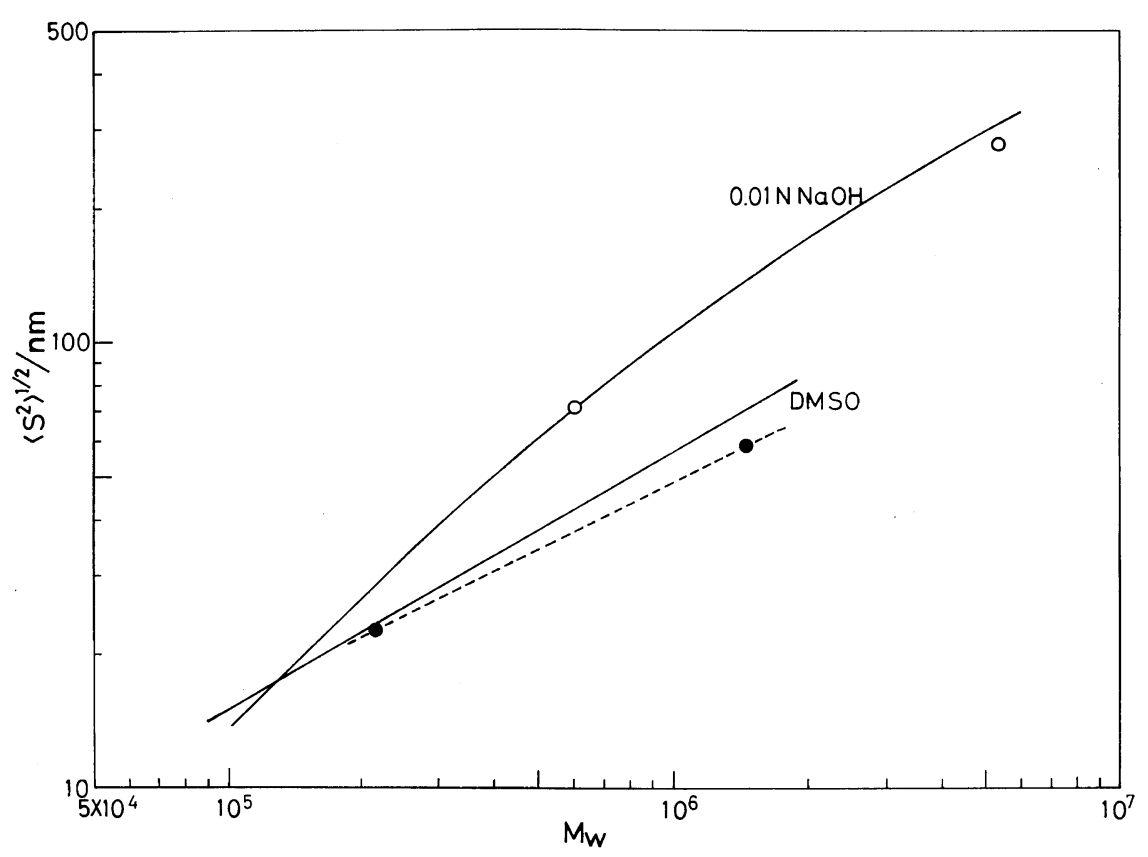

Figure 5. Comparison of the values of $\left\langle S^{2}\right\rangle^{1 / 2}$ for scleroglucan in $0.01 \mathrm{~N} \mathrm{NaOH}(\mathrm{O})$ and DMSO with the reported values ${ }^{6}$ (solid curves) for schizophyllan in the same solvents.

scleroglucan chain in DMSO appears to be a random coil with negligible excluded volume. The $A_{2}$ values for DMSO are consistent with this result (see Table I). On the other hand, the solid line for schizophyllan in DMSO has a slope of 0.58 , indicating that this polysaccharide behaves as a random coil undergoing an appreciable excludedvolume effect in DMSO.

\section{Intrinsic Viscosity}

The values of $[\eta]$ and $k^{\prime}$ (the Huggins constant) for the samples S-2 and N-2 in $0.01 N \mathrm{NaOH}$ and DMSO at $25^{\circ} \mathrm{C}$ are presented in the sixth and seventh columns of Table I. These $[\eta]$ values are plotted double-logarithmically against $M_{w}$ in Figure 6 . Here the solid curves fit the corresponding data for schizophyllan ${ }^{5,6}$; the slope of the line for $0.01 \mathrm{~N}$ $\mathrm{NaOH}$ is about 1.8 in the region of $M_{w}$ below $5 \times 10^{5}$ and about 1.1 at $M_{w} \sim 5 \times 10^{6}$, while that for DMSO is 0.69 over the entire range of $M_{w}$ indicated. The data points for $0.01 N \mathrm{NaOH}$ do not deviate much from the solid line for the same solvent, confirming that the scleroglucan trimer in $0.01 \mathrm{~N} \mathrm{NaOH}$ is rodlike. The dashed line connecting the data points for DMSO has a slope of about 0.5 .

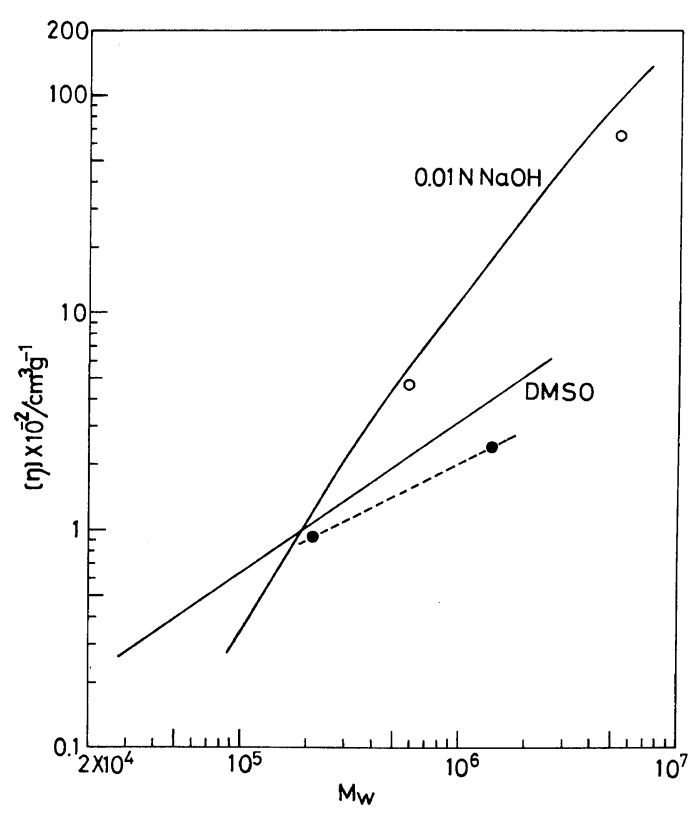

Figure 6. Comparison of the values of $[\eta]$ for scleroglucan in $0.01 \mathrm{~N} \mathrm{NaOH} \mathrm{(O)} \mathrm{and} \mathrm{DMSO} \mathrm{(O)} \mathrm{with}$ the reported values ${ }^{5,6}$ (solid curves) for schizophyllan in the same solvents. 


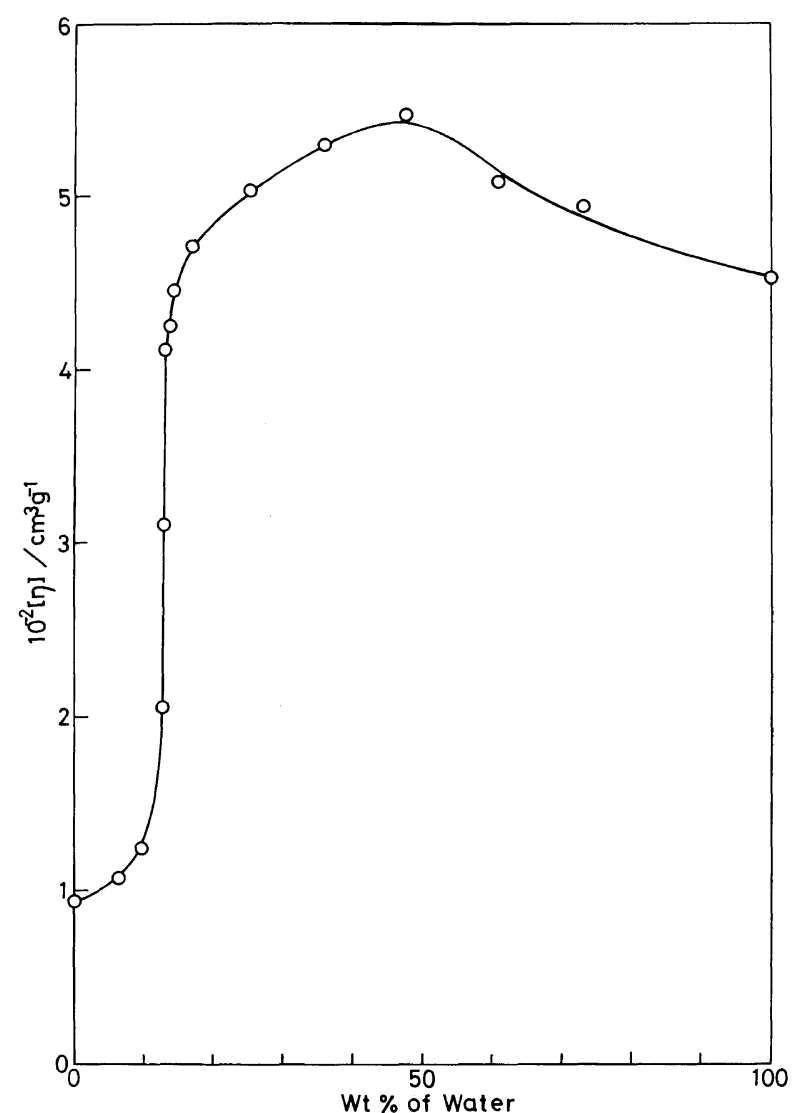

Figure 7. Composition dependence of $[\eta]$ for the scleroglucan sample S-2 in water + DMSO mixtures at $25^{\circ} \mathrm{C}$.

Table I. Results from light scattering and viscosity measurements on scleroglucan samples in $0.01 \mathrm{~N} \mathrm{NaOH}$ and DMSO at $25^{\circ} \mathrm{C}$

\begin{tabular}{|c|c|c|c|c|c|c|}
\hline \multirow{2}{*}{ Sample } & \multirow{2}{*}{ Solvent } & \multirow{2}{*}{$10^{-4} M_{w}$} & $10^{4} \mathrm{~A}_{2}$ & \multirow{2}{*}{$\frac{\left\langle S^{2}\right\rangle^{1 / 2}}{\mathrm{~nm}}$} & \multirow{2}{*}{$\frac{10^{-2}[\eta]}{\mathrm{cm}^{3} \mathrm{~g}^{-1}}$} & \multirow{2}{*}{$k^{\prime}$} \\
\hline & & & $\mathrm{cm}^{3} \mathrm{~mol} \mathrm{~g}^{-2}$ & & & \\
\hline \multirow[t]{2}{*}{$\mathrm{S}-2$} & $0.01 N \mathrm{NaOH}$ & 60.0 & 1.8 & 71.7 & 4.59 & 0.43 \\
\hline & DMSO & 21.3 & 0.7 & 22.6 & 0.932 & 0.37 \\
\hline \multirow[t]{2}{*}{$\mathrm{N}-2$} & $0.01 \mathrm{~N} \mathrm{NaOH}$ & 540 & 0.6 & 276 & 65.8 & 0.45 \\
\hline & DMSO & 143 & 0.5 & 57.7 & 2.43 & 0.34 \\
\hline
\end{tabular}

This is consistent with the finding from $\left\langle S^{2}\right\rangle$ that the scleroglucan molecule in DMSO behaves like an unperturbed flexible chain.

\section{Viscosity Behavior in Water + DMSO Mixtures.}

Figure 7 shows the composition dependence of $[\eta]$ of the sample S-2 in water+DMSO mixtures at. $25^{\circ} \mathrm{C}$. The $[\eta]$ value in pure water is approximately the same as that in $0.01 \mathrm{~N} \mathrm{NaOH}$ (see Table I). Though not shown in this paper, the value of $[\eta]$ for the sample $\mathrm{N}-2$ in pure water is also close to that in $0.01 \mathrm{~N} \mathrm{NaOH}$. These facts suggest that, as found for schizophyllan, ${ }^{6}$ the conformation of scleroglucan is essentially similar in pure water and $0.01 \mathrm{~N} \mathrm{NaOH}$. Hence, the data in Figure 7 can be taken to depict the way in which the trimer of scleroglucan in water 
dissociates to single chains as DMSO is added to the solvent. As the composition of DMSO increases, $[\eta]$ gradually increases, passes through a broad maximum, and decreases very sharply at a DMSO composition of about $87 \mathrm{wt} \%$. At present, no reasonable interpretation can be found for the appearance of the maximum, but what appears to be more significant is the fact that the DMSO composition for the abrupt drop of $[\eta]$ is exactly the same as that at which the triple helix of schizophyllan dissociates almost discontinuously to single chains. ${ }^{5}$ This agreement is another evidence for the similarity of the two glucans.

\section{DISCUSSION}

We observed that the dimensional and hydrodynamic behavior of Polytran scleroglucan in $0.01 \mathrm{~N}$ $\mathrm{NaOH}$ is very similar to that of schizophyllan in the same solvent or in pure water. This finding is consistent with the result from our chemical analysis showing that the repeat unit of scleroglucan may be considered identical with that of schizophyllan. Thus, from what we have found in recent studies $^{5,6,15}$ on schizophyllan in aqueous solutions, we may conclude that scleroglucan should exist in $0.01 N \mathrm{NaOH}$ as a rigid trimer in which the polymer chains assume a triple helical structure.

We also obtained experimental evidence indicating that scleroglucan is dispersed as a randomly coiled single chain in DMSO as is schizophyllan in the same solvent. ${ }^{5,6}$ However, there was a distinct difference in the random coils of these glucans in DMSO with respect to the dependence of $\left\langle S^{2}\right\rangle$ and [ $\eta$ ] on $M_{w}$. The random coil of scleroglucan appears to be almost in an unperturbed state, while that of schizophyllan is expanded appreciably by volume exclusion. This finding is striking, since our chemical analysis revealed no substantial difference between the repeat units of these two glucans. It rather suggests that their molecular chains differ so subtly as to prevent detection by the analytical methods employed in the present work.

We have demonstrated that practically no $\beta$-1,6linkages are present in the main chain of scleroglucan, as already found to be the case with schizophyllan. ${ }^{4}$ It is therefore reasonable to suspect that the above mentioned difference in the random coils of these glucans in DMSO should be primarily associated with their side chains. Both our analysis and those made by other workers ${ }^{2-4}$ confirm for both glucans that there is one $\beta$ 1,6-glucosidic side chain for every three $\beta$-1,3glucosidic main chain residues and that virtually no other side chains are present in the molecules. However, these analyses fail to show which of the three main chain residues in a repeat unit carries the side chain or how the side chains are distributed along the backbone chain. We conjecture that experimental exploration of this problem will provide a clue for explaining this striking difference.

The molecular model constructed in our laboratory indicates that the triple helices of $\beta-1,3-\mathrm{D}-$ glucans are determined essentially by the main chain residues. In fact, nearly the same helix pitches have been reported for schizophyllan ${ }^{6,15}$ in dilute solution, lentinan ${ }^{16}$ in the crystalline state, and curdlan ${ }^{17}$ in the crystalline state, all of which differ only in the population of $\beta$-1,6-D-glucosidic side chains relative to that of $\beta$-1,3-D-glucose residues in the main chain. Hence, scleroglucan and schizophyllan should form very similar triple helices even if there is a difference in the distribution of side chains of the two glucans. Experimental determination of the pitch of the scleroglucan triple helix in $0.01 \mathrm{~N} \mathrm{NaOH}$ will be reported later.

Acknowledgments. We are very grateful to Professor H. Fujita for his critical reading of this manuscript, valuable comments, and interest in this work. The chemical analysis was made possible through the guidance and advice of Professor A. Misaki of Osaka City University, to whom we are thankful. Thanks are also extended to MitsuiBussan Co. for the scleroglucan sample used.

\section{REFERENCES}

1. Ceca S. A. Technical Report, Nov., 1976.

2. J. Johnson, Jr., S. Kirkwood, A. Misaki, T. E. Nelson, J. V. Scletti, and F. Smith, Chem. Ind., 820 (1963).

3. S. Kikumoto, T. Miyajima, K. Kimura, S. Okubo, and N. Komatsu, J. Agric. Chem. Soc. Jpn., 45, 162 (1971).

4. K. Tabata, W. Ito, T. Kojima, S. Kawabata, and A. Misaki, Carbohydr. Res., 89, 121 (1981).

5. T. Norisuye, T. Yanaki, and H. Fujita, J. Polym. Sci., Polym. Phys. Ed., 18, 547 (1980).

6. Y. Kashiwagi, T. Norisuye, and H. Fujita, 
Macromolecules, 14, 1220 (1981).

7. S. Hakomori, J. Biochem. (Tokyo), 55, 205 (1964).

8. P. F. Fleury and J. Lange, J. Pharm. Chem., 17, 196 (1933).

9. M. Abdel-Akher, J. K. Hamilton, R. Montgomery, and F. Smith, J. Am. Chem. Soc., 74, 4970 (1952); F. Smith and J. W. Van Cleve, J. Am. Chem. Soc., 77, 3091 (1955).

10. E. T. Reese and M. Mandels, Can. J. Microbiol., 5, 173 (1959).

11. M. Somogyi, J. Biol. Chem., 195, 19 (1952); N. Nelson, J. Biol. Chem., 153, 375 (1944).
12. H. Björndal, B. Lindberg, and S. Svensson, Acta Chem. Scand., 21, 1801 (1967).

13. M. Dubois, K. A. Gilles, J. K. Hamilton, P. A. Rebers, and F. Smith, Anal. Chem., 28, 350 (1956).

14. I. Hirano, Y. Einaga, and H. Fujita, Polym. J., 11, 901 (1979).

15. T. Yanaki, T. Norisuye, and H. Fujita, Macromolecules, 13, 1462 (1980).

16. T. L. Bluhm and A. Sarko, Can. J. Chem., 55, 293 (1977).

17. Y. Deslandes, R. H. Marchessault, and A. Sarko, Macromolecules, 13, 1466 (1980). 